\title{
Design and Enactment of Dynamically Reconfigurable Bus Enhanced NoC Architecture for Emerging Digital System
}

\author{
A.Mariyammal, J. Rajprabha , A.Sountharyabanu, N.Sangavi, G.Malarvizhi
}

\begin{abstract}
The large amount imperative issue in present VLSI circuit proposes in the area and power reduction. This work proposes a new architecture which reduces an area efficiently.The reimbursement of adding a little latency, modified mutual bus as an essential element of the NoC structural design is explored. This architecture design reduces the charge of partisan a broad choice of design occurrence through specified throughput needs by minimizing the requirement of design entities in the architecture design of NoC road and rail network for the area minimization.
\end{abstract}

Keywords: Master Memory, Slave Memory, Network Interface, Switch, Processing Element.

\section{INTRODUCTION}

The Network on Chip has initiated during competent announcement to solve the growing confronts in the NoC communications. In stipulations of multiple operation with the same path through the production of collision, to assemble with the measurable strain, it deals about the structure stage confront for intrachip multistage announcement. This hybrid NoC merges and exploits with the uniform metal NoC connection and the creation of Wireless-NoC. Particularly, an aim of the inspection of contention treatment in Surface Wave Interconnects layer is measured together federal and decentres protocol. By using the combined-wire-SWI design, it eliminates congestion to the association, alleviates the formation of traffic hotspots and avoids deadlocks. The evaluation based on a cycle accurate reproduction and hardware explanation. The efficiency of power utilization is approximately $10 \mathrm{X}$ and recital is about $22 \mathrm{X}$. By means of the mixture wire SWI design for NoC, zenneck facade wave less power indulgence, elevated indication, and spread hustle and fanout potential gives to tackle the 1 towards $M$ traffic concerns efficiently. An output shows the important enhancement in terms of normal delay, drenched PIR and power utilization with a comparatively diminutive die region with verdict evaluated condition to design the architectures. It results in the high scalability of the W-SWI for the many cores era.

Here Silicon-proven design is used for a new on- network to hold up assured transfer variation multiprocessor. In this

Revised Manuscript Received on 14 August, 2019.

A. Mariyammal, Assistant Professor, Vivekananda College of Engineering for Women (Autonomous), Tiruchengode, Tamil Nadu, India. (Email: apmariyammal148@gmail.com)

J. Rajprabha, UG Scholar, ekananda College of Engineering for Women (Autonomous), Tiruchengode, Tamil Nadu, India.

A. Sountharyabanu, UG Scholar, ekananda College of Engineering for Women (Autonomous), Tiruchengode, Tamil Nadu, India

N. Sangavi, UG Scholar, ekananda College of Engineering for Women (Autonomous), Tiruchengode, Tamil Nadu, India

G. Malarvizhi, UG Scholar, ekananda College of Engineering for Women (Autonomous), Tiruchengode, Tamil Nadu, India. network they use pipelined circuit-switching come close to multiple path-setup plans below a multipath system intention. The multiple path-setup plans scheme facilitates the execution understanding for random transfer variation. The circuit switching move toward presents a guaranteed of permuted data and its flattened slide allows the benefits of stacking multi set-ups a $0.13 \mu \mathrm{m}$ through CMOS legalizes the probability and competency of planned devise. In the onchip network design we use pipelined with energetic path setup proposal with run time pathway agreement.

Before multi path complex method supporting network topology. Here topology is in three stages closed network distinct as $c(n, m, p)$. The rearrangable path is between its input and output the middle stage switching is in minimum cost with rearrangable property with three stages like setup, transfer and release. Switch by switch connection with handshakes of 1 bit for request and a 2-bit for answer. $\mathrm{R}=1$ switch request an ideal link to the corresponding downstream switch in the set phase. $R=0$ release the occupied link ans $=01$ (ack) destination is ready to receive data from the source, propagate back to the source and data transfer in progress immediately. Ans $=11$ (n ack) end-to-end flow organize while in the receipt of the circuit not organized to receive information with the concept of busy with other task. Ans=(10 back $)$ link is block due to back force control of the active path-setup. The expected results show that the proposed on-chip network achieves the diminution of silicon over head evaluated to additional devise approach.

\section{RELATED WORK}

In the prospectchip multi-processorrequisites of extensibility, resemblance and structure modularization through bus interfaces are used and it ismeasured among the key interrelate communications in the potential CMP. In this paper, by addition of minimum retort time, adapted to the collectiveinterconnection through the all design entities which is to be discovered. The interpolation of bus entity has been introduced with intrinsically competent of possible delivery of diffusion through proficient approach.The bus entity has been processed with the combination of subordinate and conventional circulation of the same data.The simulation (2) process is utilized to valuate BENoC all the way through DNUCA multiprocessor system.During the execution of a typical CMP application, the greater part of the transfer distributed with the connection

Published By:

Blue Eyes Intelligence Engineering \& Sciences Publication 
concerned point-to-point announcement. The BENoC is collected of two strongly included parts of fast execution, usage of small amount of bandwidth dedicated bus, optimized for system extensive allocation of control signals and a large throughput data communication between pairs of modules. The bus is intrinsically a solitary hop, transmit medium, BENoC has more cost than a network foundation intersect. BENoC architecture is simulated using OPNET. BENoC's bus is wormed only to hurl messages dissimilar from individualsdistributed by the network, such as control and multicast communication.This method will reduces the power consumption of an interrelation as a solitary bus contraction achieves the transmit action, as an alternative of using the multiples unicast messages in the NoC and also the system performance is improved and reduces the interconnect energy. The bus enhanced NoC architecture optimizes the communication infrastructure by combining a customized bus and a NoC. By using the bus for searching cache lines, the total interconnect energy consumption is reduced by $16 \%$ on average.

The majority of previous estimation of plump trees for Noc connection networks relies on simplified idealistic design and transfer prototype postulations, and a small number of layout analysis are obtainable to alleviate practical possibility apprehensions in nanoscale expertise. This employment intend at given that a thoroughly evaluation of physical synthesis competence of plump trees and at estimating (5) silicon attentive presentation figure to reverse interpret in the system-level presentation study. A $2 \mathrm{D}$ mesh is old as location design true for evaluation, and a $65 \mathrm{~nm}$ expertise is beleaguered by our revision. As a final point, in a challenge to moderate the accomplishment cost of $\mathrm{K}$-ary n-tree topologies, furthermore estimate a substitute unidirectional multi path connection set-up which is used to simplify the fat-tree design and to minimize the collision routine.

\section{DESIGN ENTITIES}

The SOC consists of Master, Slave00, Slave01, Slave11, Network Interface, Switch, On-Chip Peripheral Bus to transmit the data from one processor to another processor.

\section{MASTER MEMORY UNIT}

The main functions of the master unit used to give command to slave unit. The operation has to be with the use of input signals, and output signals. The input signals are the data input, data address, clock, reset, and read/write signal.

The data output signal, request and grant signal to the network interface located at the output side of the diagram as shown in figure 1.1 .

When the master unit wishes to send data to a slave unit, it sends data, address and grant signals to the Network Interface. As soon as NI receives a request (a high in grant signal) it looks up the routing table compares the source and destination address and determines the route.

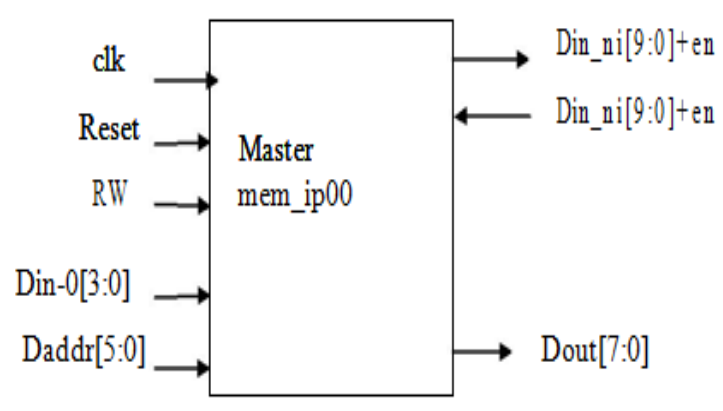

Fig 1.1 Master Unit

\begin{tabular}{|c|c|c|c|}
\hline Input & $\begin{array}{c}\text { Cloc } \\
\text { k }\end{array}$ & $\begin{array}{c}\text { Grant } \\
\text { signal }\end{array}$ & $\begin{array}{c}\text { Outpu } \\
\mathbf{t}\end{array}$ \\
\hline 1010 & $\mathbf{0}$ & $\mathbf{0}$ & $\mathbf{0 0 0 0}$ \\
\hline 1010 & $\mathbf{0}$ & $\mathbf{1}$ & $\mathbf{0 0 0 0}$ \\
\hline 1010 & $\mathbf{1}$ & $\mathbf{1}$ & $\mathbf{1 0 1 0}$ \\
\hline 1010 & $\mathbf{1}$ & $\mathbf{0}$ & $\mathbf{0 0 0 0}$ \\
\hline
\end{tabular}

Table 1.1 Representation of output generation in the Master memory unit

When a master requests a data from slave unit, it enables the grant signals and the address signal from which it requires the data. The packet generated by NI is sent to slave unit through the slave unit. The slave unit upon receiving the requested packet reads the packet and determines the route from the header. The slave NI generates a new packet with the route and data requested and sends it back to the master node.

\section{SLAVE UNITS}

The main function of slave unit used to receive the command signal from the master unit. It explains the operation in the form of input signals, and output signals. The input signals are the data input, clock, and reset signal. The request and grant signal to the network interface located at the output side of the diagram as shown in figure 1.2.

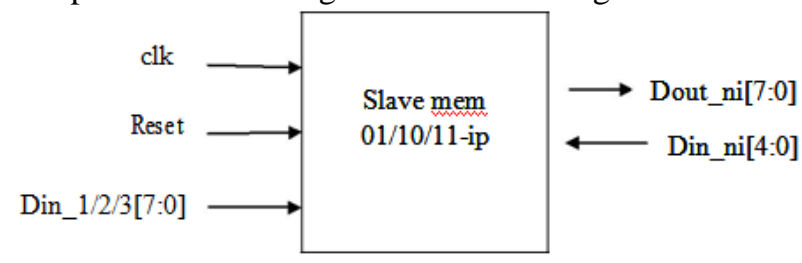

Fig 1.2 Slave Units

\section{INTEGRATION OF NOC}

The network on chip can be explained with the model of master and slave unit. Here the master is one of the processor then the slave unit is the corresponding chip such as the video, audio and data transfer IC. The communication between these devices based on the consumer requirements. The master unit used to write and read the data as per the manufacturer. This process obtained by the clock, reset, readand write signal. The slave unit has the operation of read 
the data from the master unit. The master unit gives the command to the slave unit. Based on the requirement of the master command the slave unit responds to the master. Nanometer technologies agree to incorporation of countless transistors with in a particular processor. The reception of sending data and receiving data gets from different design entity.More number of integrated processing elements with more design entities into one processor is known as the amalgamation of system. The main face up to the designer of the systems is required to conquer the process with an accurate and consistent function of the networking entities.

\section{INTEGRATION OF NOC WITH BUS BASED ARCHITECTURE\& RESULTS}

It contains the elements of master unit, slave unit, buses, network interface and switches. It occupies less amount of silicon area and numbers of communication devices are less it solves traffic during the delivery of data. It combines both features of NOC with bus based approach. The bus unit is the extra component in this diagram as compared to the integration NOC architecture.

Bus unit operation organized by control and data lines. Control lines used to give the signal to apply for the acknowledgements. Data lines represents to carry the information stuck between the master entities to any of the slave entity.

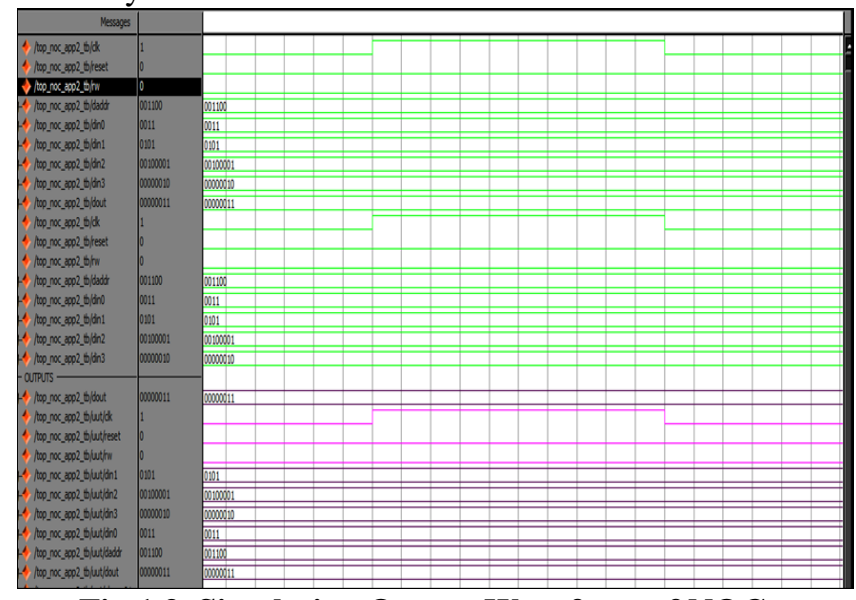

Fig 1.3. Simulation Output Waveform of NOC

\section{SYNTHESIS REPORTS}

Processor can be designed using different design methods. The most common method in practice today is known as register transfer level design. The synthesis result shows the RTL design and corresponding area occupation in table form 1.2 and 1.3.

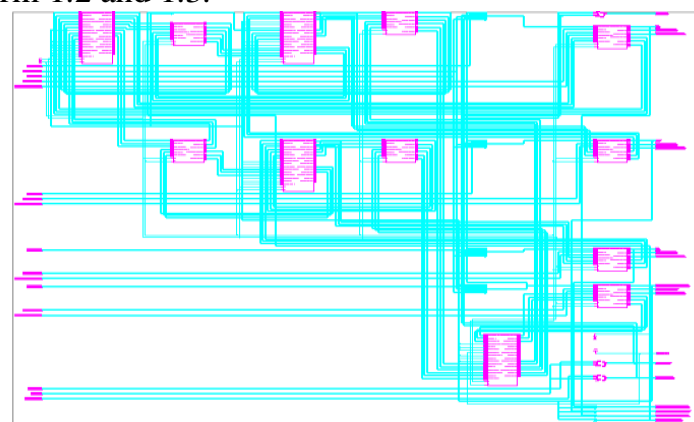

Fig 1.4 RTL view of integration on NOC model

\begin{tabular}{|c|c|c|}
\hline \multicolumn{3}{|c|}{ Device Utifization Sumnary (estinated values) } \\
\hline Logic Utilization & Used & Available \\
\hline Numben of Silices & 4168 & 4656 \\
\hline Number of Sice Flip Flops & 4252 & 9312 \\
\hline Number of 4 rout LUTs & 6799 & 9312 \\
\hline Number of banded $108 \mathrm{~B}$ & 190 & 190 \\
\hline Number of GCKKs & 1 & 24 \\
\hline
\end{tabular}

Table 1.2 Device utilization summary for Integration on NOC Model

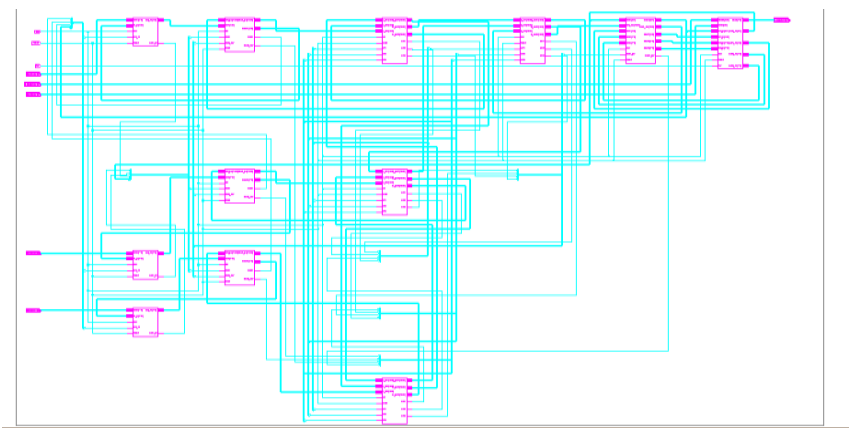

Fig 1.5 RTL view of integration on NOC with Bus Based model

\begin{tabular}{|c|c|c|c|}
\hline \multirow[b]{2}{*}{ Logic Utilization } & \multicolumn{2}{|c|}{ Device Utitization Summary } & \multirow[b]{2}{*}{ Utilization } \\
\hline & Used & Available & \\
\hline Nunber of Slice Flip Flops & 1,152 & 9,312 & $12 \%$ \\
\hline Nunber of 4 input LUTs & 1,427 & 9,312 & $15 \%$ \\
\hline \multicolumn{4}{|l|}{ Logic Distribution } \\
\hline Number of occupied Sices & 1.213 & 4.656 & $26 \%$ \\
\hline Number of Slices containing only relaled bogc & 1,213 & 1.213 & $100 \%$ \\
\hline Nunber of Slices contaning unelaled logic & 0 & 1.213 & $0 \%$ \\
\hline Total Number of 4 input LUTs & 2069 & 9,312 & $22 \%$ \\
\hline Nunber used as logic & 1,427 & & \\
\hline Number used for Dual Pout RAMs & 618 & & \\
\hline Nurber used as 16x1 RAMs & 24 & & \\
\hline Number of bonded $1088 s$ & 41 & 190 & $21 \%$ \\
\hline 108 Flp flops & 8 & & \\
\hline Number of GCLKs & 1 & 24 & $4 \%$ \\
\hline
\end{tabular}

Table 1.3 Device utilization summary for Integration on NOCwith Bus Based Model

The integration NOC architecture with bus based design contains less amount of area when compared to the integration of NOC architectureas represented in the comparison result fig 1.6.

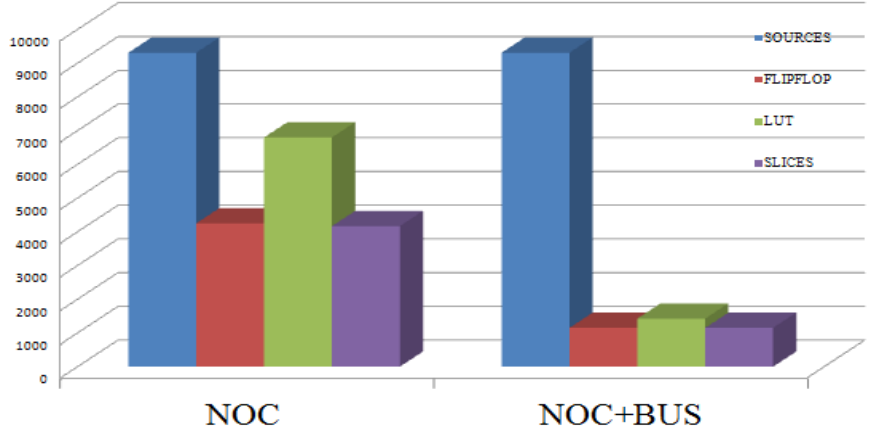

Fig 1.6 Comparison of NOC and NOC with Bus Based systems 


\section{DESIGN AND ENACTMENT OF DYNAMICALLY RECONFIGURABLE BUS ENHANCED NOC ARCHITECTURE FOR EMERGING DIGITAL SYSTEM}

\section{CONCLUSION}

The integration of NOC architecture design made more amount of area which engages more digital workings. The more no of digital devices generate the gate-level counts in an increased order, which escort to diminish the speed of the circuit and devour much quantity of power in the SOC plane integrations. In previous chapter the table shows that integration of NOC with bus based model contains less no of digital devices makes the gate-level counts in a smaller amount of number, which directs to amplify the speed of the circuit and devours less power in the SOC stage integrations. Hence the integration NOC architecture with bus based design contains less amount of area when compared to the integration of NOC architecture. The Integration of NOC with bus based architecture be able to scheming the reconfiguration organizer and also functional in active varying surroundings.

\section{REFERENCES}

1. Phi Hung Pham, Junyoung Song, Jongsun Park, and Chulwoo Kim (2013), 'Design and Implementation of an On-Chip Permutation Network for Multiprocessor System-On-Chip' in proc. IEEE transactions on very large scale integration (VLSI) systems, vol. 21, no. 1.

2. Alessandro Meroni, Vincenzo Rana, Marco Santambrogio, Francesco Bruschi, (2009) 'Design of Communication Infrastructures for Reconfigurable Systems'in Proc.IEEE Int. Symp. Circuits Syst. (ISCAS), Volume 36, pp 291-307.

3. Benes (1965), Mathematical Theory of Connecting Networks and Tele-phone Traffic. New York: Academic Press.

4. Bertozzi and Benini (2004). Xpipes: a network-on-chip architecture for gigascale systems-on-chip. Circuits and Systems Magazine, IEEE, 4(2):18-31.

5. Bjerregaard and Mahadevan (2006), 'A Survey of research and practices of Network on- chip' ACM Computing Surveys (CSUR), vol. 38 Issue 1.

6. Bobda and Ahmadinia (2005), 'Dynamic interconnection of reconfigurable modules on reconfigurable devices' IEEE Design\&Test of Computes, vol. 22, pp. 443-451.

7. Bobda, Ahmadinia, Majer, Teich, Fekete, and van der Veen (2005), 'DyNOC: A dynamic infrastructure for communication in dynamically reconfigurable devices' pp. $153-158$.

8. Dally and Towles (2004), Principles and Practices of Interconnection Networks: San Francisco.

9. Gaughan and Yalamanchili (1995), 'A family of faulttolerant routing protocols for direct multiprocessor networks' IEEE Trans. Parallel Distrib. Syst., vol. 6, no. 5, pp. 482-497.

10. ITRS (2007), 'The international technology roadmap for semiconductors 2007 edition: system drivers', Available: http://www.itrs.net, pp. 7.

11. Ludovici, Gilabert, Medardoni, Gomez, Gomez, Lopez, Gaydadjiev, and Bertozzi , (2009), 'Assessing fat-tree topologies for regular network-on-chip design under nanoscale technology constraints' in Proc. Design, Autom. Test Euro. Conf. Exhib. (DATE), pp. 562-565.

12. Mak, Sedcole, Cheung, and Luk (2005), 'On-fpga communication architectures and design factors'.

13. Michael, Nikolov, Tang, Suh, and Batten (2011), 'Analysis of application-aware on-chip routing under traffic uncertainty' in Proc. IEEE/ACM Int. Symp. Netw. Chip (NOCS), pp. 9-16.

14. Moore (1965), 'Cramming more components onto integrated circuits' Electronics, vol.38,no. 8.
15. Moussa, Baghdadi, and Jezequel, (2008) , 'Binary de Bruijn on-chip network for a flexible multiprocessor LDPC decoder' in Proc. ACM/ IEEE Design Autom. Conf. (DAC), pp. 429-434.

16. Neeb, and Thul (2005), 'Network-on-chip-centric approach to interleaving in high throughput channel decoders' in Proc. IEEE Int. Symp. Circuits Syst. (ISCAS), pp. 1766-1769.

17. Pham, Mau, and Kim (2009), 'A 64-PE folded-torus intra-chip communication fabric for guaranteed throughput in network-on-chip based applications' in Proc. IEEE Custom Integr. Circuits Conf. (CICC), pp. 645-648.

18. Pham, Park, Mau, and Kim(2010), 'Design and implementation of backtracking wave-pipeline switch to support guaranteed throughput in network-on-chip' IEEE Trans. Very Large Scale Integr. (VLSI) Syst., 10.1109/TVLSI.

19. Pionteck, Koch, and Albrecht (2007), 'Applying partial reconfiguration to networks-on-chips'.

20. Simone Corbetta (2005), 'Network on chip eases ip connect' Embedded Systems Europe, Matr.: 708346

21. Thilo Pionteck, Carsten Albrecht, Roman Koch, Erik Maehle Michael Hubner, Jurgen Becker (2007), 'Communication Architectures for Dynamically Reconfigurable FPGA Designs’ IEEE VOL1-4244-09101.

22. Vangal, Howard, Ruhl, Dighe, Wilson, Tschanz, Finan, Singh, Jacob, Jain, Erraguntla, Roberts, Hoskote, Borkar, and Borkar (2008), 'An 80-tile sub-100-w Teraflops processor in 65-nm CMOS' IEEE J. Solid-State Circuits, vol. 43, no. 1, pp. 29-41.

23. Xiang and Liu (2008), 'Research on building block design and verification for NoC' Master Thesis, Zhejiang University, Hangzhou, China, pp. 35. 